\title{
Yuri Manin on Biomolecules as Quantum Computers
}

\author{
Terry B. Bollinger (1) 1 \\ ${ }^{1}$ Apabistia Research, Ashburn, VA, USA 20147
}

(Incepted 5 February 2021; Published 5 February 2021; Tweaked 2021-07-07.21:47)*

In 1980, Russian mathematician Yuri Manin published Computable and Uncomputable. On pages 14 and 15 of his introduction, Manin suggests that "Molecular biology furnishes examples of the behavior of natural (not engineered by humans) systems which we have to describe in terms initially devised for discrete automata." Manin then describes the remarkable energy efficiency of naturally occurring biomolecular processes such as DNA replication. He proposes modeling such behaviors in terms of unitary rotations in a finite-dimensional Hilbert space. The decomposition of such systems then corresponds to the tensor product decomposition of the state space, that is, to quantum entanglement. Manin's initial focus on biological molecules as examples of highly energy-efficient quantum automata is unique among quantum computing's founding figures since both he and other early leaders quickly moved to the then-new and exciting concept of von Neumann automata. The von Neumann formalism reinterpreted molecular quantum computing in terms of qubits, which made it possible to imagine the power of quantum computing as not much more than a superposition of virtual binary computers. This paper provides the original excerpt of Manin's molecular computing argument. A valuable feature of Manin's first assessment is its openness to new formalisms that avoid employing classical machines of extraordinary and complexity and precision as the assumed base states of quantum superpositions.

DOI: https://doi.org/10.48034/20210205
This is about: Quantum Computation, Thermodynamics of computation

Published by Apabistia Press under the Creative Commons Attribution 4.0 International License. You are free to redistribute this article as you choose, provided only that you preserve the author attribution, article title, journal citation, and DOI.

${ }^{*}$ This document uses Kenobi versioning. The online name always points to the most recent document version, while obl, ob2,... suffixes indicate obsolete releases of the document. 
Molecular biology furnishes examples of the behaviour of natural (not engineered by humans) systems which we have to describe in terms initially devised for discrete automata. Figure 2 (next page) is a scheme of RNA translation and protein synthesis. ${ }^{3}$ It does look somewhat like a drawing of a Turing machine copying information from one tape to another.

A classical continuously evolving system governed by differential equations can imitate a discrete automaton only if its phase space is extremely elaborate: it must include many stability domains, or attractors, separated by low energy barriers. The input of a program creates a labyrinthine system of passages in these barriers creating a path for a trajectory that approximates the discrete process of computation. As a physical system, a computer must be extremely unstable because a change in one bit of input generally leads to a totally different computation. But the computation itself as a physical evolution must be very stable: jumping over a closed barrier as a result of physical fluctuations must be highly improbable. It is well known that these requirements (combined with inherent speed restrictions and the growth of dissipated energy with the growth of complexity) doomed the development of mechanical computers. Nevertheless, we believe that "genetic automata" can be described in such mechanical terms. One of the well-known problems to which such a description leads is a picture of DNA replication. Replication of the double helix of a bacterial chromosome involves the uncoiling of about 300,000 turns accompanied by an intricate set of highly specific chemical reactions.

Perhaps, for a better understanding of this phenomenon, we need a mathematical theory of quantum automata. Such a theory would provide us with mathematical models of deterministic processes with quite unusual properties. One reason for this is that the quantum state space has far greater capacity than the classical one: for a classical system with $N$ states, its quantum version allowing superposition (entanglement) accommodates $c^{N}$ states. When we join two classical systems, their number of states $N_{1}$ and $N_{2}$ are multiplied, and in the quantum case we get exponential growth $c^{N_{1} N_{2}}$.

These crude estimates show that the quantum behavior of the system might be much more complex than its classical simulation. In particular, since there is no unique decomposition of a quantum system into its constituent parts, a state of the quantum automaton can be considered in many ways as a state of various virtual classical automata. Cf. the following instructive comment at the end of the article by R. P. Poplavskii, "Thermodynamical models of information processing" (in Russian), Uspekhi Fizicheskikh Nauk, 115:3 (1975), 465-501: "The quantum-mechanical computation of one molecule of methane requires $10^{42}$ grid

\footnotetext{
${ }^{3}$ The figure presented here was redrawn for this edition by M. Gelfand and O. Khleborodova, to whom I owe my deepest gratitude.
} 


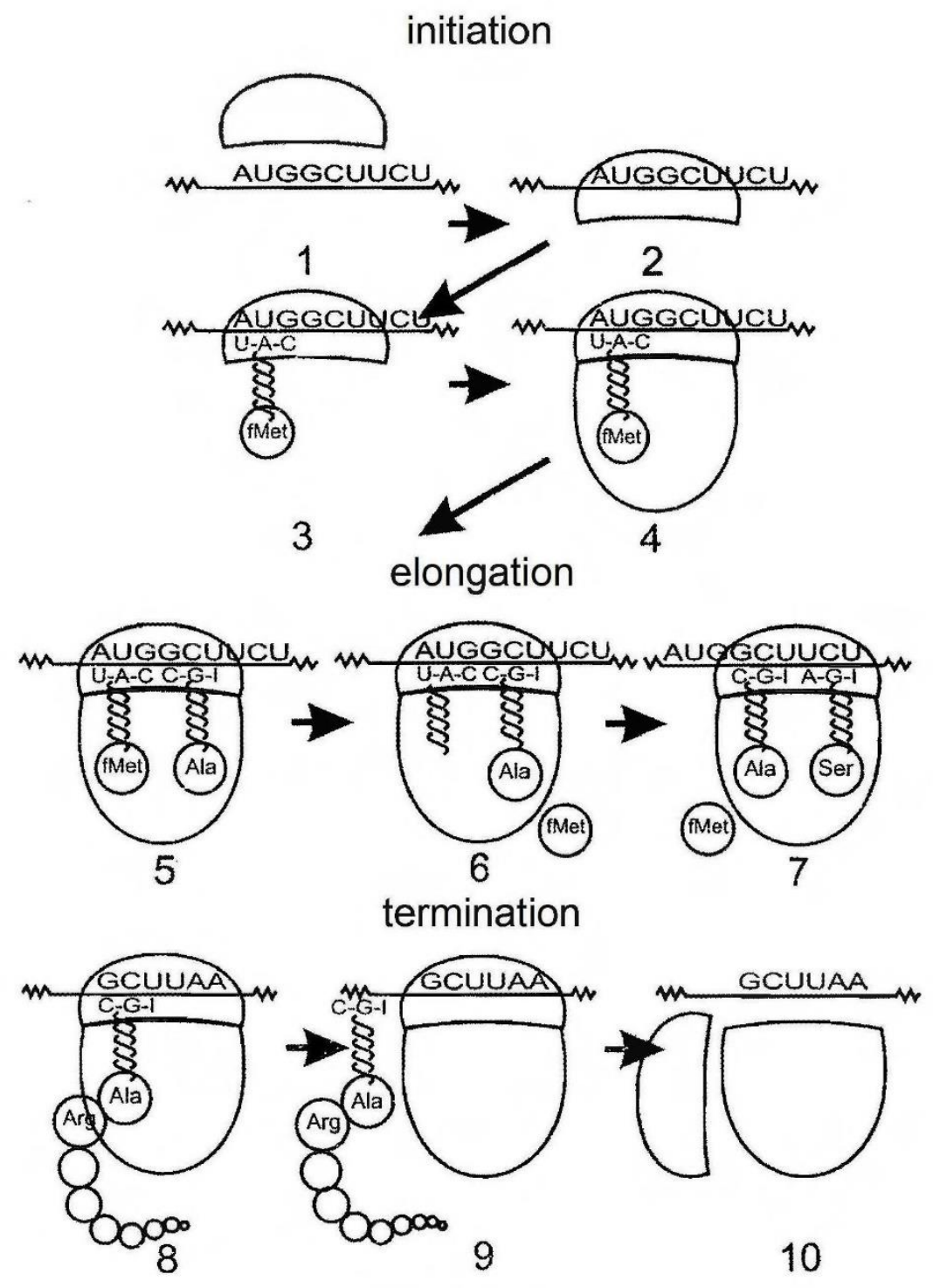

Figure 2

points. Assuming that at each point we have to perform only 10 elementary operations, and that the computation is performed at the extremely low temperature $T=3 \cdot 10^{-3} \mathrm{~K}$, we would still have to use all the energy produced on Earth during the last century."

The first difficulty we must overcome is the choice of the correct balance between the mathematical and the physical principles. The quantum automaton has to be an abstract one: its mathematical model must appeal only to the general principles of quantum physics, without prescribing a physical implementation. Then the model of evolution is the unitary rotation in a finite dimensional Hilbert space, and the decomposition of the system into its virtual parts corresponds to the tensor product decomposition of the state space ("quantum entanglement"). Somewhere in this picture we must accommodate interaction, which is described by density matrices and probabilities. 